\title{
Relación Entre el Peso y la Talla de Madres y Recién Nacidos de Alto Riesgo Obstétrico
}

\author{
Doctora Eva Awad Z* Doctor Alfonso Jubiz H. **
}

\section{INTRODUCCION}

La nutrición de la madre durante el embarazo es un factor importante para el adecuado desarrollo del feto.

Si la embarazada recibe una alimentación balanceada antes y durante la gestación, el recién nacido tendrá buenas reservas nutricionales y ella, estará en mejores condiciones para la lactancia.

Una manera indirecta para determinar el grado de nutrición de la madre durante el embarazo, es el control del peso a lo largo de la atención prenatal.

Factores como la talla y la edad parece que influyen sobre el peso de la mujer adulta no embarazada, pero el peso durante la gestación no está modificado por las condiciones anteriores. La ganancia de peso en este período es diferente para cada embarazada y muchos son los factores que pueden influir para el incremento semanal o mensual (2).

Tradicionalmente se han aceptado cifras promedios y así podemos decir, que una ganancia de 10 kilos (21 libras) es adecuada, 7 kilos (15 libras) es deficiente $y$ el aumento es muy deficiente cuando es de $51 / 2$ kilos (12 libras). Chesley, citado por Eastman y Hellman considera

\footnotetext{
* Profesora II - Enfermera

** Profesor Titular III
}

como normal un peso de 24 libras durante todo el embarazo y recomienda una dieta de 1.800 calorías (1).

Entre nosotros no existen estudios que relacionen las variables peso y talla materna con las del recién nacido.

Como hipótesis del presente trabajo se consideró que el peso y la talla materna tienen una influencia directa sobre las mismas variables en el recién nacido.

\section{Objetivos}

1. Determinar la relación existente entre el peso de la madre en kilos, registrado en la última consulta prenatal y el peso en gramos del recién nacido.

2. Determinar la relación existente entre la talla de la madre medida en centímetros y la del recién nacido.

\section{Material y Métodos}

El universo está constituido por 217 embarazadas que consultaron al Departamento de Obstetricia y Ginecología y que fueron atendidas durante su control prenatal en el programa de riesgo perinatal durante el primer semestre del presente año.

Se tuvo en cuenta el peso materno registrado durante la última consulta prenatal, casi siempre en la semana previa al parto. 
Para consignar el peso se tuvieron en cuenta las siguientes condiciones:

1. Balanza detecto calibrada.

2. Control del peso entre las 7 y las 8 de la mañana.

3. Embarazada sin zapatos.

4. El peso siempre fue tomado por la misma persona (Enfermera Auxiliar). La talla se anotó en centímetros y el dato se tomó con el tallímetro que trae incorporado la balanza.

Los datos del recién nacido se tomaron inmediatamente después del parto por personal de enfermería con entrenamiento adecuado y utilizando el mismo sistema para pesar y medir los niños.

No se hizo selección en relación con los factores de riesgo, y se incluyeron dos diabéticas. Se buscó el coeficiente de correlación entre el peso y la talla de las madres y los hijos respectivamente.

\section{Resultados}

\section{Peso Materno}

El peso promedio de las madres fue de 62 kilos y 500 gramos, con una desviación estándar de 10.30 kilos.

\section{Peso de los recién nacidos}

El peso promedio de los recién nacidos fue de 3.100 gramos y la desviación estándar (D.S) de 396.15 gramos.

\section{Talla de las Madres}

La talla promedio encontrada fue de 152 centímetros con una D.S. de 5.71 cent ímetros.

\section{Talla de los recién nacidos}

Los recién nacidos presentaron una talla promedio de 48.22 centímetros.

Al analizar el coeficiente de correlación del peso, se encontró que es positi- vo entre el peso de la madre $y$ el del recién nacido. $(r=0.3368$ y p.L 0.001)

En el análisis de la talla materna no se encontró ninguna correlación con la del recién nacido.

\section{Discusión}

El análisis de los resultados nos indica que el peso de la madre registrado una semana antes del parto, guarda estrecha relación con el peso del recién nacido y podemos esperar, que entre más pese la madre, mayor será el peso del niño. Consideramos que este hallazgo nos obliga a permanecer vigilantes durante la consulta prenatal para que la embarazada tenga un incremento adecuado de peso. Si éste es exagerado, no solamente aumenta la incidencia de la pre-eclampsia, sino que también puede ser causa de distocia por la macrosomía fetal. Lo mismo podemos decir del incremento inadecuado en relación con el recién nacido de bajo peso.

Consideramos de importancia el desarrollo de estudios que nos permitan conocer la distribución de estas variables en una población normal.

\section{Resumen}

Se analizaron el peso y la talla de 217 embarazadas de alto riesgo que consultaron al Departamento de Obstetricia $y$ Ginecología durante el primer semestre del presente año. El peso promedio de las madres fue de 62 kilos 500 gramos $y$ el de los recién nacidos de 3.100 gramos. La D.S. del peso materno fue de 10.30 kilos y la de los niños de 396.15 gramos.

Al analizar el coeficiente de correlación $(r=0.3368)$ se encontró positivo entre el peso de la madre y el del recién nacido p. $\angle 0.001$, por lo tanto, es de esperar, que entre más pese la madre mavor será el peso del niño. 
En el análisis de la talla materna se encontró que el promedio fue de 152 cent ímetros y el de los recién nacidos de 48.22. La D.S. de la talla de las embarazadas fue de 5.71 y de 2.16 centímetros para los niños. No se encontró ninguna correlación entre estos datos.

\section{RELATION BETWEEN WEIGHT AND HEIGHT OF MOTHERS AND NEWBORNS WITH HEIGH OBSTETRIC RISK}

\section{Summary}

An analysis was made on the weight and height of 217 pregnant women with high obstetric risk, who attended the Ginecology and Obstetrics Department during the first half of this year. The mothers' average weight was 62 kilos 500 grams and that of the newborns was 3.100 grams. The mothers' D.S. weight was 10.30 kilos and that of the newborns was 396.15 grams.

After the analysis, the analogy coefficient $(3=0.3368)$ appeared positive between the weight of the mother. and the newborn p. 0.001 . Therefore, it is evident that the higher the weight of the mother is, the higher will be the weight of the baby.
The height analysis showed an' average of 152 centimeters for the mother and 48.22 for the newborns. The height D.S. of the pregnants was 5.71 and 2.16 centimeters for the babies. data.

No analogy was found among these

\section{Conclusiones}

1. Se encontró una correlación altamente positiva entre el peso de la madre y el del recién nacido. (p. $\angle 0.001)$.

2. No se encontró ninguna correlación entre la talla de la gestante y su recién nacido.

\section{Bibliografía}

1. EASTMAN, N.J. and HELLMAN, L.M. Williams Obstetrics. N. York: AppletonCentury-Crofts. 12 th. Ed. 1961 pp. 193, 235-237.

2. NSP-3 Evaluación del peso de la embarazada. Instituto de Nutrición de Centro América y Panamá. INCAP No. 153, junio, 1961. 